\title{
Efecto sedativo de la asociación xilazina-morfina en caninos
}

\author{
Sedative effects of the association between xylazine and morphine in canine
}

\author{
H Bustamante, M Werner \\ Instituto de Ciencias Clínicas Veterinarias, Universidad Austral de Chile, Valdivia, Chile.
}

\begin{abstract}
SUMMARY
The aim of this study was to evaluate the sedative and adverse effects produced by the endovenous combination of xylazine and morphine administered to dogs. Fourty dogs of different breed, size and age were used. Each animal received $0.4 \mathrm{mg} / \mathrm{kg}$ of xylazine and $0.4 \mathrm{mg} / \mathrm{kg}$ of morphine i.v. Sedation was evaluated using the punctuation score described by Young y col (1990). The scores were obtained before the administration and 5, 10 and 20 minutes after administration. Heart rate, respiratory rate and the presentation of side effects during the evaluation time were also recorded. The results obtained show also that the scores in the analyzed variables presented significant differences $(\mathrm{P}<0.05)$, indicating a marked sedative effect produced by the association between xylazine and morphine. In the same way, there was a significant decrease $(\mathrm{P}<0.05)$ in heart rate and respiratory rate. Side effects that appeared during the study were excitement and respiratory effort. It can be concluded that the endovenous administration of xylazine and morphine combination produced significant sedation in dogs and side effects as excitement and minor respiratory effort.
\end{abstract}

Palabras clave: xilazina, morfina, caninos, sedación.

Key words: xylazine, morphine, canines, sedation.

\section{INTRODUCCIÓN}

La sedación se define como un estado caracterizado por la depresión del sistema nervioso central (SNC) acompañado por somnolencia (Thurmon y col 1996). Dentro de los grupos de fármacos con acción sedativa más utilizados en caninos se encuentran los derivados fenotiazínicos, aquellos derivados de las butiferonas, las benzodiazepinas, el grupo de fármacos alfa 2 adrenérgicos y el grupo de narcóticos (Flores y Cattaneo 2000).

Flores y Cattaneo (2001) clasifican a los agonistas de los receptores alfa 2 adrenérgicos como compuestos con poderosos efectos sedantes y analgésicos, pero con muchos efectos colaterales, que actúan estimulando los receptores alfa 2 adrenérgicos. Sumano y Ocampo (1997) indican que el efecto clínico de esta acción farmacológica son sedación, analgesia y relajación muscular. Cullen (1999) indica que sus efectos sedativos son producto de la estimulación de receptores alfa 2 a nivel cerebral, además, la acción analgésica se produce por estimulación de diversos tipos de receptores, ubicados principalmente a nivel espinal y cerebral. Su mecanismo de acción está dado por producir una depresión del SNC mediante estimulación de los adrenoceptores alfa 2 presinápticos tanto a nivel central como periférico, reduciendo la liberación de noradrenalina, lo que se traduce en una disminución de la actividad simpática del SNC y una disminución de catecolaminas circulantes (Muir y col 2001). Xilazina fue reportada como el primer

Aceptado: 20.05.2009.

hbustamante@uach.cl agonista alfa 2 en ser usado como sedante y analgésico en medicina veterinaria (Thurmon y col 1996). Sus principales efectos fisiológicos, dejando de lado aquellos relacionados con el SNC, ocurren a nivel de los sistemas cardiovascular y respiratorio. Existen médicos veterinarios que evitan su utilización debido a sus propiedades cardiodepresoras y arritmogénicas (Thurmon y col 1996); estos efectos son según Hall y col (2001) modificados por acción del sistema nervioso autónomo, produciéndose, según Cullen (1999), una disminución en la actividad simpática y un aumento del tono parasimpático a nivel central. Maze y Tranquilli (1991) indican que posterior a la administración endovenosa de xilazina se produce bradicardia y un corto período de hipertensión, seguido por una prolongada hipotensión. A nivel respiratorio, England y Clarke (1989) señalan que la administración de agonistas alfaadrenérgicos produce una marcada disminución en la frecuencia respiratoria. De esta forma, xilazina induce una depresión de los centros respiratorios, lo que resulta en una reducción de la frecuencia respiratoria (Hall y col 2001), sin alterar el pH, $\mathrm{pO}_{2}, \mathrm{pCO}_{2}$ arteriales (Klide y col 1975). Del mismo modo, Haskins y col (1986) reportan una disminución de la frecuencia respiratoria y de la ventilación/minuto en perros posterior a la administración de xilazina. Dentro de otros efectos se incluyen alteraciones a nivel de la motilidad gástrica, prolongándose el tiempo de tránsito gastrointestinal en perros (Hsu y McNeel 1983).

Según Thurmon y col (1996), el término opioide es utilizado para referirse a todos los compuestos tanto exógenos como sintéticos que se unen a subpoblaciones específicas de receptores opiáceos. El mecanismo de acción de los fármacos opiáceos está mediado por la 
proteína G. Existen diversos tipos de proteínas G; las que se relacionan con los opioides se caracterizan por producir efectos inhibitorios a nivel neuronal (Chahl 1996). Esta actividad inhibitoria se caracteriza por: a) inhibición de la actividad bioeléctrica y b) inhibición de la liberación del neurotransmisor en que esa neurona se ha especializado, sea cual fuere su naturaleza (Flórez 1992). Según Hug y col (1981), morfina en pequeñas dosis analgésicas ha logrado popularidad como suplemento anestésico en la medicina veterinaria, siendo el prototipo de un opiáceo agonista. Su principal efecto farmacológico es producir analgesia, induciendo un marcado y rápido aumento en la síntesis de serotonina, lo que se correlaciona con su efecto analgésico (Godefroy y col 1980). Los efectos de la morfina se pueden clasificar como una mezcla de excitación y depresión del SNC, ejerciendo efectos simpaticomiméticos y parasimpaticomiméticos y de liberación de histamina (Sumano y Ocampo 1997). Dentro de sus efectos más importantes se incluyen depresión respiratoria producto de acción a nivel de los centros respiratorios centrales, produciendo una disminución del volumen respiratorio minuto (Finck y col 1977). Bailey y Stanley (1998) sugieren que estas alteraciones respiratorias representan el principal inconveniente de la administración de opiáceos, siendo la depresión dosisdependiente. La principal causa de aparición de alteraciones respiratorias sería una interferencia con los centros respiratorios ubicados en la protuberancia y la médula espinal, los cuales regulan la ritmicidad respiratoria. De la misma forma se produce una estimulación del centro del vómito, produciéndose emesis, siendo las especies más sensibles los caninos y felinos. A nivel cardiaco, Bailey y Stanley (1998) señalan que con excepción de meperidina todos los opiáceos estimulantes del receptor $\mu$ suelen producir reducciones de la frecuencia cardiaca. Además, la administración de morfina puede producir en perros vasoconstricción coronaria y una disminución de la presión arterial (Plumb 1999), pudiendo esto deberse a una marcada liberación de histamina, siendo la especie canina especialmente sensible a los efectos de ésta (Hall y col 2001). Según Bailey y Stanley (1998), este aumento en las concentraciones plasmáticas de histamina posterior a la administración de morfina provoca dilatación de las arteriolas terminales, siendo estas reacciones más frecuentes posterior a la administración endovenosa (Hall y col 2001).

Diversos factores hacen que la utilización de la asociación entre un opiáceo y un alfa 2 agonista sea considerada importante al momento de intentar obtener variables grados de sedación en medicina veterinaria. Thurmon y col (1996) señalan que tanto los receptores opiáceos como los alfa 2 adrenérgicos son encontrados en regiones similares del SNC e incluso en las mismas poblaciones neuronales. De la misma forma, comparten similares mecanismos moleculares posterior a la unión fármaco-receptor, en la cual, una vez activada la proteína $\mathrm{G}$, se abren canales de potasio en la membrana neuronal, lo que hace que la neurona se hiperpolarice, no respondiendo a los estímulos excitatorios (Paddleford 1999). Esta asociación en dosis apropiadas puede incrementar el grado de sedación sin causar alteraciones significativas a nivel cardiaco y respiratorio (England y Watts 1997). De igual forma, diversos autores han investigado el grado de sedación obtenido en perros a partir de la combinación de un opiáceo y un alfa 2 agonista administrados principalmente por vía intramuscular (Bartram y col 1993, Bartram y col 1994, Ko y col 1996, Robinson y col 2001). Dentro de las principales conclusiones obtenidas a partir de los estudios señalados destacan la buena capacidad sedativa de las combinaciones, adosada a una buena analgesia, lo cual resultó en la posibilidad de llevar a cabo procedimientos menores dentro de la práctica veterinaria.

El objetivo de este estudio es evaluar los efectos sedativos y los posibles efectos colaterales presentados posteriores a la administración endovenosa de la asociación de xilazina-morfina en caninos.

\section{MATERIAL Y MÉTODOS}

Se utilizaron 40 perros que ingresaron para consulta de rutina a la Clínica de Pequeños Animales del Hospital Veterinario de la Facultad de Ciencias Veterinarias de la Universidad Austral de Chile durante el año 2002. Los animales correspondieron a individuos de diferentes edades, razas y tamaños, alcanzando un peso promedio de $15,4 \pm 6,05 \mathrm{~kg}$. Cada paciente fue pesado y depilado en la parte craneal del miembro anterior izquierdo para la posterior colocación de un catéter endovenoso $21 \mathrm{G}$ en la vena cefálica izquierda. A cada animal se le administró una dosis de $0,4 \mathrm{mg} / \mathrm{kg}$ de peso vivo de xilazina y $0,4 \mathrm{mg} /$ $\mathrm{kg}$ de morfina por vía endovenosa (Doham y col 2001). La determinación del efecto sedativo de la combinación farmacológica fue realizada utilizando la pauta de puntuación desarrollada por Young y col (1990) (cuadro 1). La determinación del puntaje de los animales se llevó a cabo previo a la administración de la combinación. Posterior a ello, los animales fueron llevados a un lugar tranquilo donde permanecieron por espacio de 5 minutos. Se repitió la medición de la puntuación a los 5, 10 y 20 minutos posterior a que fueron traídos para la evaluación. De igual manera, las frecuencias cardiaca y respiratoria fueron determinadas por espacio de 20 minutos mediante la auscultación directa del tórax. Igualmente se llevó un registro de los posibles efectos colaterales que pudieran aparecer durante el estudio.

Los datos obtenidos fueron registrados en una planilla confeccionada para tal fin, identificando el número de animal, edad, sexo y raza. Se utilizaron métodos de estadística descriptiva y análisis de varianza no paramétrico de Friedman y el test de comparación múltiple de Dunn. Se utilizaron los programas computacionales Microsoft $₫$ Excel y Graph Pad Prism 3.0 (Graph Pad Software, La Joya, California, USA). 
Cuadro 1. Sistema de puntuación desarrollado por Young y col (1990) usado para la determinación del efecto sedativo de la combinación farmacológica en perros.

Punctuation system developed by Young et al (1990) used for the determination of sedative effect of the pharmacologic combination in dogs.

\begin{tabular}{|c|c|c|}
\hline PARÁMETRO & RESPUESTA & PUNTAJE \\
\hline \multirow[t]{5}{*}{ Postura espontánea } & De pie & 0 \\
\hline & Atáxico pero de pie & 1 \\
\hline & $\begin{array}{l}\text { Decúbito pero puede } \\
\text { incorporarse }\end{array}$ & 2 \\
\hline & $\begin{array}{l}\text { Decúbito con dificultad } \\
\text { para incorporarse }\end{array}$ & 3 \\
\hline & Decúbito permanente & 4 \\
\hline \multirow[t]{4}{*}{ Colocación en decúbito lateral } & Fuerte resistencia & 0 \\
\hline & Modesta resistencia & 1 \\
\hline & Leve resistencia & 2 \\
\hline & Sin resistencia & 3 \\
\hline \multirow[t]{5}{*}{ Respuesta al estímulo sonoro } & Se incorpora & 0 \\
\hline & Escucha y se mueve & 1 \\
\hline & $\begin{array}{l}\text { Escucha y mueve las } \\
\text { orejas }\end{array}$ & 2 \\
\hline & No percibe & 3 \\
\hline & No responde & 4 \\
\hline \multirow[t]{3}{*}{ Relajación mandibular } & Pobre & 0 \\
\hline & Leve & 1 \\
\hline & Buena & 2 \\
\hline \multirow[t]{4}{*}{ Actitud general } & Excitable & 0 \\
\hline & Despierto y normal & 1 \\
\hline & Tranquilo & 2 \\
\hline & Deprimido & 3 \\
\hline \multirow[t]{4}{*}{ Reflejo pedal } & Normal & 0 \\
\hline & Respuesta moderada & 1 \\
\hline & Respuesta leve & 2 \\
\hline & Sin respuesta & 3 \\
\hline
\end{tabular}

\section{RESULTADOS}

\section{GRADO DE SEDACIÓN}

Previo a la administración de la combinación de xilazina y morfina, el puntaje obtenido para el parámetro de postura espontánea fue en promedio de $0,8 \pm 0,91$. Cinco minutos posterior a la administración, el puntaje aumentó en forma significativa $(\mathrm{P}<0,05)$, para llegar a un valor de 3,5 $\pm 0,98$. Los más altos puntajes fueron obtenidos 10 minutos posterior a la administración de los fármacos, con valores de 4,00 $\pm 0,8$ y a los 20 minutos, con valores de $4,00 \pm 0,72$. Entre los tiempos cinco, 10 y 20 postadministración no se presentaron diferencias significativas $(\mathrm{P}>0,05)$ (cuadro 2).

Previo a la administración, el puntaje promedio obtenido para la colocación en decúbito lateral fue de $0,25 \pm 0,59$. Los puntajes resultantes posterior a la administración de xilazina y morfina fueron los siguientes: a los 5 minutos, $3,00 \pm 0,46$, a los 10 minutos de $3,00 \pm 0,16$ y a los 20 minutos, 3,00 \pm 0,49 (cuadro 2). El puntaje promedio previo presenta diferencias significativas $(\mathrm{P}<0,05)$ con los tiempos 5, 10 y 20 . De la misma forma, entre estas tres últimas mediciones, las diferencias encontradas no son significativas $(\mathrm{P}>0,05)$ (cuadro 2 ).

Relativo a la respuesta a estímulos sonoros, ningún animal recibió puntos al inicio del experimento, es decir, en la medición previa a la administración de xilazina y morfina. A los 5 minutos, el promedio de los puntajes aumenta en forma significativa $(\mathrm{P}<0,05)$, para llegar a un valor de $3,00 \pm 0,92 ;$ a partir de esta medición, a los 10 minutos los valores aumentan en forma no significativa $(\mathrm{P}>0,05)$ para llegar a $3,00 \pm 0,85$. Veinte minutos posterior a la administración, los valores alcanzan a 3,00 $\pm 0,74$ (cuadro 2). De esta forma, se presentan diferencias significativas $(\mathrm{P}<0,05)$ entre la medición previa y cada una de las mediciones posteriores. Entre estas últimas, no se presentan diferencias significativas $(\mathrm{P}>0,05)$ (cuadro 2$)$.

Previo al inicio del experimento, los animales no presentaron ningún tipo de relajación mandibular, lo que se reflejó en un promedio de puntajes de 0 . Cinco minutos posterior a la administración de la asociación entre xilazina y morfina, los animales aumentaron su puntaje en forma significativa $(\mathrm{P}<0,05)$, para llegar a un promedio de 2,00 $\pm 0,44$. A los 10 minutos, el promedio del puntaje obtenido aumentó en forma significativa $(\mathrm{P}<0,05)$ en comparación con la primera valoración, alcanzando puntajes de 2,00 $\pm 0,16$. Posteriormente, a los 20 minutos, el puntaje se mantiene constante, llegando a 2,00 $\pm 0,3$, siendo este valor significativamente mayor $(\mathrm{P}<0,05)$ en comparación con la valoración previa al inicio del experimento. De la misma forma, entre las tres últimas mediciones las diferencias observadas son de carácter no significativo $(\mathrm{P}>0,05)$ (cuadro 2).

Los valores promedio del parámetro actitud general, previos a la administración de la asociación entre xilazina y morfina, fueron de $1,00 \pm 0,39$. La medición realizada 5 minutos después muestra que los valores aumentan significativamente $(\mathrm{P}<0,05)$, llegando a 2,00 $\pm 0,51$. Los valores aumentan levemente y en forma no significativa $(\mathrm{P}>0,05)$ a los 10 minutos, alcanzando valores de 3,00 $\pm 0,55$. La misma tendencia continúa hacia los 20 minutos posteriores a la administración, alcanzando valores promedio de 3,00 $\pm 0,38$. Las diferencias encontradas entre estas tres últimas mediciones resultaron ser no significativas $(\mathrm{P}>0,05)$ (cuadro 2).

Los puntajes obtenidos para evaluar la presencia de reflejo flexor inmediatamente antes de la administración de los fármacos a evaluar obtuvieron en promedio un valor de 0 . Cinco minutos posterior a la administración, los valores aumentan significativamente $(\mathrm{P}<0,05)$, para llegar a un valor promedio de 3,00 $\pm 0,67$. De igual manera, se presentan diferencias significativas $(\mathrm{P}<0,05)$ entre la valoración previa y aquella realizada a los 10 minutos de 
Cuadro 2. Promedio, desviación estándar (D.E.) y rango de los puntajes indicadores de sedación con la asociación xilazina-morfina en caninos $(\mathrm{n}=40)$.

Mean, standard deviation (S.D.) and range in sedation scores with the association between xylazine and morfine in dogs $(n=40)$.

\begin{tabular}{|c|c|c|c|c|c|}
\hline PARÁMETRO & PREVIO & $5 \mathrm{MIN}$ & $10 \mathrm{MIN}$ & $20 \mathrm{MIN}$ & $\mathrm{P}$ \\
\hline Postura espontánea & $\begin{array}{c}0,8 \pm 0,91^{\mathrm{a}} \\
0-2\end{array}$ & $\begin{array}{c}3,5 \pm 0,98^{b} \\
0-4\end{array}$ & $\begin{array}{c}4 \pm 0,8^{b} \\
0-4\end{array}$ & $\begin{array}{c}4 \pm 0,72^{b} \\
1-4\end{array}$ & $\mathrm{P}<0,0001$ \\
\hline Colocación en decúbito lateral & $\begin{array}{c}0,25 \pm 0,59^{\mathrm{a}} \\
0-2\end{array}$ & $\begin{array}{c}3 \pm 0,46^{b} \\
2-3\end{array}$ & $\begin{array}{c}3 \pm 0,16^{b} \\
2-3\end{array}$ & $\begin{array}{c}3 \pm 0,49^{b} \\
1-3\end{array}$ & $\mathrm{P}<0,0001$ \\
\hline Respuesta al estímulo sonoro & $0 \pm 0^{\mathrm{a}}$ & $\begin{array}{c}3 \pm 0,92^{b} \\
1-4\end{array}$ & $\begin{array}{c}3 \pm 0,85^{b} \\
1-4\end{array}$ & $\begin{array}{c}3 \pm 0,74^{\mathrm{b}} \\
2-4\end{array}$ & $\mathrm{P}<0,0001$ \\
\hline Relajación mandibular & $0 \pm 0^{\mathrm{a}}$ & $\begin{array}{c}2 \pm 0,44^{b} \\
1-2\end{array}$ & $\begin{array}{c}2 \pm 0,16^{\mathrm{b}} \\
1-2\end{array}$ & $\begin{array}{c}2 \pm 0,3^{\mathrm{b}} \\
1-2\end{array}$ & $\mathrm{P}<0,0001$ \\
\hline Actitud general & $\begin{array}{c}1 \pm 0,39^{\mathrm{a}} \\
0-3\end{array}$ & $\begin{array}{c}2 \pm 0,51^{b} \\
2-3\end{array}$ & $\begin{array}{c}3 \pm 0,55^{b} \\
1-3\end{array}$ & $\begin{array}{c}3 \pm 0,38^{b} \\
2-3\end{array}$ & $\mathrm{P}<0,0001$ \\
\hline Reflejo flexor & $0 \pm 0^{\mathrm{a}}$ & $\begin{array}{c}3 \pm 0,67^{\mathrm{b}} \\
1-3\end{array}$ & $\begin{array}{c}3 \pm 0,36^{\mathrm{b}} \\
2-3\end{array}$ & $\begin{array}{c}3 \pm 0,38^{b} \\
2-3\end{array}$ & $\mathrm{P}<0,0001$ \\
\hline Total & $\begin{array}{c}2 \pm 1,18^{\mathrm{a}} \\
0-6\end{array}$ & $\begin{array}{c}15,38 \pm 2,1^{\mathrm{b}} \\
8-19\end{array}$ & $\begin{array}{c}17,05 \pm 1,88^{\mathrm{c}} \\
9-19\end{array}$ & $\begin{array}{c}17 \pm 1,67^{c} \\
13-19\end{array}$ & $\mathrm{P}<0,0001$ \\
\hline
\end{tabular}

Letras diferentes en sentido horizontal indican diferencias significativas entre periodos.

llevada a cabo la administración, la cual obtiene un puntaje promedio de 3,00 $\pm 0,36$. La valoración realizada a los 20 minutos obtiene un promedio de puntaje de 3,00 $\pm 0,38$, siendo este valor significativamente diferente $(\mathrm{P}<0,05)$ a aquel obtenido previo a la administración de la asociación entre xilazina y morfina. De igual manera, las diferencias encontradas entre las últimas tres mediciones no fueron significativas $(\mathrm{P}<0,05)$ (cuadro 2$)$.

\section{FRECUENCIA CARDIACA Y FRECUENCIA RESPIRATORIA}

La constante fisiológica frecuencia cardiaca presentó al inicio del experimento un valor promedio de 101,98 \pm 17,47 latidos por minuto. Estos valores iniciales descienden marcadamente durante los 5 minutos posteriores a la administración de la combinación entre xilazina y morfina, llegando a un valor mínimo de 46,68 \pm 7,28 latidos por minuto a los 20 minutos postadministración (figura 1). Se producen diferencias significativas $(\mathrm{P}<0,05)$ entre la medición previa y cada una de las mediciones posteriores hasta el final del experimento. Referente a la frecuencia respiratoria, al inicio del experimento los animales presentaban una frecuencia de 27,83 $\pm 11,78$ ciclos por minuto. Posterior a la administración, la frecuencia disminuye para alcanzar un valor de 15,35 \pm 4,22 ciclos por minuto, permaneciendo constante hasta el final del experimento, en el cual el valor promedio es de 17,23 \pm 4,19 (figura 1). Se producen diferencias significativas $(\mathrm{P}<0,05)$ entre la medición previa y cada una de las mediciones posteriores hasta el final del experimento.

\section{PRESENTACIÓN DE EFECTOS COLATERALES}

Durante el desarrollo del experimento el principal efecto colateral presentado por los animales evaluados fue excitación, caracterizada por ladridos y andar descoordinado. Este período de excitación se presentó en 17/40 animales $(35 \%)$, teniendo una duración de aproximadamente 10 segundos. De la misma forma 4/40 (10\%) de los animales presentó dificultad respiratoria caracterizada por la presentación de jadeos y esfuerzo abdominal, alcanzando una duración de aproximadamente 30 segundos.

\section{DISCUSIÓN}

En el presente estudio fue hipotetizado que la asociación entre xilazina y morfina produciría una potenciación de los efectos analgésicos de ambos fármacos administrados en forma individual. De igual manera fue evaluada la presentación de posibles efectos adversos posterior a la administración de la combinación sedativa. Los efectos sedativos de la administración de xilazina han sido ampliamente descritos en la literatura, presentando resultados variables, dependiendo de la vía de administración, considerándose su efecto sedativo dosisdependiente (Pypendop y Verstegen 1998). Igualmente, Tyner y col (1997) realizaron un estudio comparativo de los efectos sedativos de medetomidina y xilazina administradas por vía intramuscular y endovenosa en caninos. Los resultados de sedación para xilazina administrada a una dosis de $1,1 \mathrm{mg} / \mathrm{kg}$ fueron catalogados como efectivos y seguros.

La sedación obtenida posterior a la administración de la asociación entre xilazina y morfina en perros puede ser considerada como excelente, esto, basándose en los puntajes promedios obtenidos dentro de cada categoría evaluada. Los resultados obtenidos en este estudio concuerdan con los encontrados en otras asociaciones entre agonistas alfa 2 y opiáceos, principalmente con la utilización de medetomidina, ya sea con butorfanol o fentanilo (Kuo y Keegan 2004, England y Clarke 1989) 


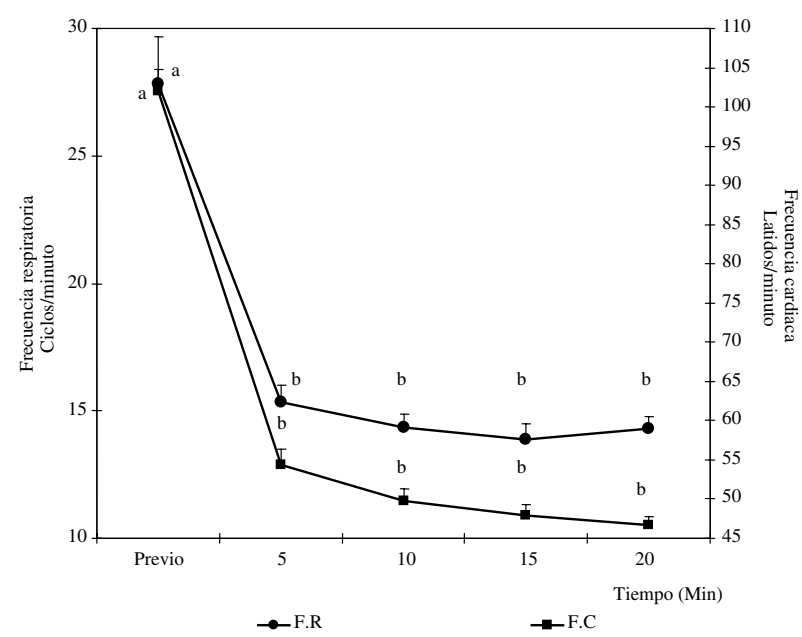

Figura 1. Promedio y desviación estándar (D.E.) de las mediciones de frecuencia cardiaca y respiratoria en caninos sedados con la combinación farmacológica xilazina-morfina $(n=40)$. Letras diferentes en sentido horizontal indican diferencias significativas entre periodos para cada variable.

Mean and standard deviation (S.D.) of heart and respiratory rate in dogs sedated with a xylazine-morfine association $(n=40)$.

o dexmedetomidina y medetomidina en combinación con buprinorfina y butorfanol (Leppänen y col 2006). La principal diferencia entre estos estudios y el aquí realizado es la utilización de agentes alfa 2 adrenérgicos de mayor potencia que xilazina. Este fármaco es el único disponible para ser utilizado en medicina veterinaria en el país y su uso se encuentra ampliamente difundido en la práctica. Monteiro y col (2008) evaluaron los efectos sedativos de la combinación entre una dosis baja de xilazina y metadona, concluyendo que dicho protocolo produjo mejores grados de sedación y analgesia al ser comparado con el grupo que sólo recibió xilazina.

Referente al parámetro postura espontánea, se observa que posterior a la administración endovenosa de los fármacos los puntajes aumentan significativamente $(\mathrm{P}<0,05)$ a los 5 minutos postadministración (cuadro 2), encontrándose los animales en decúbito. Esto concuerda con lo señalado por England y Watts (1997), quienes administrando una asociación entre romifidina y butorfanol por vía endovenosa obtienen tiempos al decúbito de 1,8 $\pm 0,3$ minutos. Esto puede explicarse por la mayor afinidad por los receptores alfa 2 que posee romifidina y por la mayor potencia absoluta que posee butorfanol, un opiáceo con actividad agonista-antagonista 4 a 7 veces más potente que la morfina (Branson y Gross 2001). El tiempo para la adopción del decúbito es similar a lo descrito por Kramer y col (1996), quienes posterior a la administración de la asociación entre xilazina y metadona describen rangos de 1-1,5 minutos. De igual forma, los resultados obtenidos son menores a los resultantes de la administración en solitario de xilazina, en dosis de 1,1 $\mathrm{mg} / \mathrm{kg}$ por vía endovenosa, con la cual Tyner y col (1997) reportan tiempos de 5,0 \pm 8,0 minutos. De igual manera, Jarvis y England (1991), administrando xilazina por vía intramuscular en dosis de $2,2 \mathrm{mg} / \mathrm{kg}$ de peso vivo, logran un tiempo de adopción del decúbito lateral de aproximadamente 10 minutos, tiempo superior al reportado en este experimento. Ko y col (1996), administrando medetomidina y una asociación entre medetomidina y butorfanol por vía intramuscular, reportan que no se producen diferencias significativas en el tiempo necesario para adoptar el decúbito lateral, lo que difiere de lo encontrado en estudios como el de Tomizawa y col (1997), los cuales indican que la adición de un opiáceo a la asociación reduce los tiempos necesarios para efectuar procedimientos que requieran de sedación. Este efecto visto al administrar fármacos por vía intramuscular podría deberse a que los fármacos inadvertidamente hayan sido depositados entre fascias musculares retardando la aparición de su efecto clínico (Ko y col 1996).

El mayor puntaje promedio para postura espontánea se produce a los 10 minutos postadministración (cuadro 2). Estos resultados concuerdan con lo señalado por Kramer y col (1996), quienes indican que la administración de la asociación entre xilazina y metadona resultó en una sedación máxima a los 30 minutos postadministración, permaneciendo los animales sedados por 120 a 180 minutos, resultados que de igual manera concuerdan con lo señalado por otros autores que utilizan la misma combinación para obtener sedación en perros (Erhardt y col 1988). De igual forma, los resultados obtenidos en este estudio concuerdan con lo reportado por England y Watts (1997), quienes posterior a la administración endovenosa de romifídina y butorfanol describen diferencias significativas en el grado de sedación a los 10 minutos postadministración, aunque el mayor puntaje obtenido por los autores señalados anteriormente se produce a los 30 minutos postadministración. De igual forma, los autores mencionan que la duración de la sedación obtenida alcanzó a 90,5 \pm 7,6 minutos, tiempo superior al encontrado posterior a la administración de xilazina y morfina. Arbeiter y col (1972), utilizando dosis de 3 a $5 \mathrm{mg} / \mathrm{kg}$ de peso vivo de xilazina por vía endovenosa reportan que el tiempo para el decúbito lateral varió entre 5 y 15 minutos, siendo la duración del efecto sedativo de alrededor de 50 minutos. Diversos autores señalan que la profundidad y el tiempo de la sedación pueden ser aumentados incorporando un opiáceo al protocolo farmacológico (Clarke y England 1989, England y Clarke 1989).

El efecto de menor duración en la acción sedativa encontrado posterior a la administración de xilazina y morfina podría deberse a la menor afinidad por receptores alfa 2 que presenta xilazina. Este efecto de menor duración se encuentra reportado en equinos por England y col (1992), quienes señalan que romifidina es un potente agonista de los receptores alfa 2 y que se caracteriza por poseer un efecto residual de mayor duración que xilazina y detomidina. Xilazina ejerce su efecto sedativo a nivel del SNC, activando o estimulando los receptores alfa 2 
adrenérgicos, lo cual disminuye la descarga simpática y reduce la liberación de noradrenalina (Gross 2001). Muir (1991) indica que esta estimulación produce una hiperpolarización neuronal e inhibe la liberación y almacenamiento de noradrenalina y dopamina.

Referente al grado de relajación muscular, evaluada mediante el grado de apertura mandibular (cuadro 2), los resultados no concuerdan con aquellos señalados por England y col (1996), quienes indican que posterior a la administración de romifidina por sí sola en perros no se produjo suficiente relajación muscular como para lograr una completa relajación mandibular. Esto no concuerda con lo señalado por Bartram y col (1994), quienes posterior a la administración de medetomidina y butorfanol por vía intramuscular no presentaron problemas en el posicionamiento de los animales para procedimientos radiográficos, generalmente en decúbito dorsal, con los miembros flexionados o extendidos, siendo estos efectos producto del buen grado de relajación muscular obtenido. Xilazina, según Gross (2001), produce relajación musculoesquelética mediante la inhibición de la transmisión de impulsos interneurales a nivel central en el SNC, siendo esta capacidad, según Muir (1991), más pronunciada que cualquier otro fármaco sedativo o hipnótico utilizado en medicina veterinaria. Los opiáceos por su parte, según Bailey y col (2000), pueden aumentar el tono muscular e incluso causar rigidez muscular, mecanismo que aún no se encuentra claramente descrito. Algunos autores como Jurna y col (1972) y Freye y Kuschinsky (1976) han sugerido que los opiáceos producen rigidez alterando las concentraciones de dopamina a nivel cerebral. Incluso Havemann y Kuschinsky (1981) señalan que la estimulación de interneuronas GABAérgicas puede encontrarse relacionada con la rigidez muscular producida posterior a la administración de algún opiáceo.

El grado de analgesia, evaluado mediante la respuesta a la estimulación del reflejo flexor, presentó diferencias significativas $(\mathrm{P}<0,05)$ entre los puntajes previos y posteriores a la administración de la combinación evaluada. Cinco minutos posterior a la administración los puntajes aumentan significativamente $(\mathrm{P}<0,05)$, para continuar aumentando hasta los 10 minutos. A los 20 minutos de iniciada la experiencia los valores disminuyen en forma no significativa $(\mathrm{P}>0,05)$, en comparación con el valor anterior. Clarke y England (1989), realizando comparaciones entre diferentes dosis de medetomidina y xilazina por vía intramuscular, reportan una disminución de la respuesta a la estimulación del reflejo flexor, siendo esta disminución de menor intensidad y duración en aquellos animales administrados con xilazina. Estos resultados concuerdan con los señalados por Jarvis y England (1991), quienes indican una reducción de la respuesta a la estimulación del reflejo flexor en un $78 \%$ de perros posterior a la administración de $2 \mathrm{mg} / \mathrm{kg}$ de xilazina por vía intramuscular. De igual forma, Tyner y col (1997) reportan aumentos en la respuesta al estímulo del reflejo flexor de hasta un $66 \%$ posterior a la administración de diferentes dosis de medetomidina y xilazina tanto por vía intramuscular como endovenosa, no encontrando diferencias significativas $(\mathrm{P}<0,05)$ entre ambos tratamientos. Kramer y col (1996) señalan que los grados de analgesia obtenidos posterior a la administración de xilazina y metadona fueron más uniformes, profundos y de mayor duración que los obtenidos con la administración de medetomidina solamente. Esto puede ser explicado por el efecto analgésico de metadona, la cual, según Lascelles (2000), es muy similar a morfina, asociándose a un menor grado de sedación y a menor presentación de efectos colaterales, con tiempos de acción de 2 a 3 horas en perros. Referente a la administración de la asociación entre un alfa 2 y un opiáceo, Muir y col (1999) señalan que posterior a la administración intramuscular de medetomidina y butorfanol en perros previo a cirugía los puntajes obtenidos para determinación del grado de analgesia indicaron menores signos de dolor 10 a 15 minutos posterior a la administración. Esto concuerda con lo descrito en trabajos anteriores utilizando asociaciones entre alfa 2 adrenérgicos y opiáceos (Bartram y col 1993, 1994, Robinson y col 2001). Este tipo de asociaciones según Cullen (1999) producen, además de un confiable grado de sedación, un importante grado de analgesia, lo cual cobra importancia a la hora de tratar con animales agresivos y para realizar procedimientos dolorosos menores.

En relación a la disminución significativa en la frecuencia cardiaca posterior a la administración de la asociación entre xilazina y morfina $(\mathrm{P}<0,05)$, los resultados no coinciden con los señalados por Kramer y col (1996), quienes describen un pequeño aumento en la frecuencia cardiaca posterior a la administración de la asociación, para posteriormente disminuir significativamente alcanzando niveles menores a los previos al experimento. Los resultados obtenidos en este estudio concuerdan en mayor medida con los obtenidos por diversos autores utilizando diversas combinaciones entre alfa 2 adrenérgicos y opiáceos (Clarke y England 1989, England y Clarke 1989, Bartram y col 1994, England y Watts 1997, Robinson y col 2001, Bustamante y Jones 2007), quienes reportan importantes disminuciones de la frecuencia cardiaca posterior a la administración de las diferentes asociaciones, las cuales se mantienen por hasta 60 minutos.

Referente a la frecuencia respiratoria, en que se observa una reducción del 55,15\% respecto de los valores previos, Kramer y col (1996) indican una disminución en la frecuencia dentro de los primeros 15 minutos, lo que coincide con lo encontrado en el presente estudio, en donde la frecuencia respiratoria disminuye significativamente $(\mathrm{P}<0,05)$ (figura 1$)$. De igual forma, los resultados concuerdan con lo encontrado por otros autores (Clarke y England 1989, England y Clarke 1989, Bartram y col 1994, England y Watts 1997, Robinson y col 2001), 
quienes describen reducciones entre el $31 \%$ y $62 \%$. Estas diferencias estarían dadas según Robinson y col (2001) por la adición de opiáceos de diferente potencia relativa. La depresión respiratoria producida por los opiáceos, en particular los fármacos $\mu$-específicos, se encuentra mediada por una disminución neuronal en la médula espinal $\mathrm{al} \mathrm{CO}_{2}$ y una lenta respuesta del centro respiratorio, la cual es dependiente de la potencia relativa del fármaco opiáceo utilizado (Lascelles 2000).

Los principales efectos colaterales que se presentaron durante el desarrollo del ensayo clínico fueron excitación $(35 \%)$ y dificultad respiratoria (10\%). Estos resultados concuerdan con lo señalado por Bartram y col (1994) y Muir y col (1999), quienes utilizando una combinación entre medetomidina y butorfanol encuentran un mínimo número de reacciones adversas o complicaciones, siendo las principales tremores musculares y excitación. Estos resultados también concuerdan con los reportados en estudios anteriores. Según Muir (2002), altas dosis de opiáceos pueden producir nerviosismo, agitación, aumento de la actividad y euforia dentro de las principales alteraciones a nivel del SNC. La excitación se encuentra generalmente asociada a la utilización de estos fármacos en felinos, especialmente al ser administrados por vía endovenosa (Lascelles 2000). De la misma forma, el mismo autor señala a la dificultad respiratoria como otra complicación de la utilización de los opiáceos, la cual generalmente se presenta como "jadeos", la que se encontraría relacionada con una alteración del centro termorregulador del hipotálamo e incluso asociada a liberación de histamina.

Del presente estudio se puede concluir que la administración endovenosa de la asociación entre xilazina y morfina en dosis de $0,4 \mathrm{mg} / \mathrm{kg}$ y $0,4 \mathrm{mg} / \mathrm{kg}$ respectivamente, produjo significativa sedación y leves efectos colaterales adversos en caninos.

\section{RESUMEN}

El objetivo del presente estudio fue evaluar los efectos sedativos y efectos colaterales presentados posterior a la administración endovenosa de la asociación entre xilazina y morfina en perros. Cuarenta perros de diferentes razas, tamaños y edades ingresados para consulta de rutina en el Hospital Veterinario de la Universidad Austral de Chile durante el año 2002 fueron utilizados. Cada animal recibió $0,4 \mathrm{mg} / \mathrm{kg}$ de xilazina y $0,4 \mathrm{mg} / \mathrm{kg}$ por vía endovenosa. Los puntajes indicadores de sedación fueron evaluados utilizando una escala de puntajes descrita por Young y col (1990). Los puntajes fueron evaluados previo a la administración de los fármacos y 5, 10 y 20 minutos posteriores a la administración de ellos. De igual forma, fueron evaluados durante este tiempo frecuencia cardiaca, frecuencia respiratoria y efectos colaterales. Los resultados indican que los puntajes de las variables analizadas aumentaron significativamente $(\mathrm{P}<0,05)$, lo que refleja un marcado efecto sedativo dado por la asociación farmacológica. De la misma forma, se determinó una disminución significativa posterior a la administración en los valores de frecuencia cardiaca y respiratoria. Durante el tiempo de evaluación se presentaron efectos colaterales caracterizados principalmente por excitación y dificultad respiratoria. Se puede concluir que la administración endovenosa de la asociación entre xilazina y morfina produjo significativa sedación y efectos colaterales como excitación y dificultad respiratoria leve en caninos.

\section{REFERENCIAS}

Arbeiter K, H Szekely, D Lorin. 1972. Results of a 5-year trial of Bay-Va 1470 (Rompun ${ }^{\circledR}$ ) in the dog and cat. Veterinary Medical Review, $3,248-258$.

Bailey PL, TH Stanley. 1998. Anestésicos opiáceos intravenosos. En: Miller RD (ed). Anestesia. Hardcourt Brace. Madrid. España.

Bailey PL, TD Egan, TH Stanley. 2000. Intravenous opioid anesthetics. In: Miller RD (ed). Anesthesia. Churchill Livingstone, Philadelphia, USA.

Bartram DH, LE Young, MJ Diamond, AS Gregg, RS Jones. 1993. Effects of combinations of medetomidine/pethidine when used for sedation and pre-anaesthetic medication in dogs. J Small Anim Pract 34, 554-558.

Bartram DH, MJ Diamond, AS Tute, AW Trafford, RS Jones. 1994. Use of medetomidine and butorphanol for sedation in dogs. J Small Anim Pract 35, 495-498.

Branson KR, ME Gross. 2001. Opioid agonist and antagonist. In: Adams HR (ed). Veterinary Pharmacology and Therapeutics. Iowa State University Press, Ames, Iowa, USA.

Bustamante H, RS Jones. 2007. Efectos cardiorrespiratorios de la administración de xilazina-morfina en perros anestesiados con pentobarbital sódico. Arch Med Vet 39, 215-222.

Chahl LA. 1996. Opioids-Mechanisms of action. Austr Prescr 19, 66-67.

Clarke KW, GC England. 1989. Medetomidine, a new sedative-analgesic for use in the dog and its reversal by atipamezole. J Small Anim Pract 30, 343-348.

Cullen LK. 1999. Xylazine and medetomidine in small animals: these drugs should be used carefully. Aust Vet J 77, 722-723.

Doham JR, LA Cohn, HE Durham, B Szladovits. 2001. Cardiorespiratory effects of intravenous morphine, xilazine and atropine in dogs. Procc Ann Mtg Am Coll Vet Anes New Orleans, LA, USA.

England GCW, KW Clarke. 1989. The use of medetomidine/fentanyl combinations in dogs. Acta Vet Scand Suppl 85, 179-186.

England GCW, KW Clarke, L Goossens. 1992. A comparison of the sedative effects of three $\alpha_{2}$-adrenoceptor agonists (romifidine, detomidine and xylazine) in the horse. $J$ Vet Pharmacol Therap $15,194-201$.

England GCW, TE Flack, E Hollingworth, R Hammond. 1996. Sedative effects of romifidine in the dog. J Small Anim Pract 37, 19-25.

England GCW, N Watts. 1997. Effect of romifidine and romifidinebutorphanol for sedation in dogs. J Small Anim Pract 38, 561-564.

Erhardt W, J Haberstroh, M Schindele, B Niehaus, KP Vick, G Blumel. 1988. The principle of "balanced anesthesia" in high risk canine patients. Tierärztl Prax 16, 179-185.

Finck AD, BA Berkowitz, BS Hempstead, SH Hgai. 1977. Pharmacokinetics of morphine: effects of hypercarbia on serum and brain morphine concentrations in the dog. Anesthesiology 47, 407-412.

Flores E, G Cattaneo. 2000. Técnicas anestésicas inyectables de uso actual. I.- Premedicación y Sedación. Monografías Med Vet 20, 34-48.

Flores E, G Cattaneo. 2001. Técnicas anestésicas inyectables de uso actual. II.- Anestésicos inyectables. Monografías Med Vet 21, 40-54.

Flórez J. 1992. Fármacos analgésicos opiáceos. En: Flórez J (ed). Farmacología Humana. Masson-Salvat, Barcelona, España.

Freye E, K Kuschinsky. 1976. Effects of fentanyl and droperidol on the dopamine metabolism of the rat striatum. Pharmacology 14, 1.

Godefroy F, J Weil-Fugazza, D Coudert, JM Besson. 1980. Effect of acute administration of morphine on newly synthesized 5-hydroxytryptamine in spinal cord of the rat. J Pharmacol Exp Ther 199, 415-422.

Gross ME. 2001. Tranquilizers, $\alpha_{2}$-adrenergic agonists, and related agents. In: Adams HR (ed). Veterinary Pharmacology and Therapeutics. Iowa State University Press/Ames, Iowa, USA.

Hall LW, KW Clarke, CM Trim. 2001. Veterinary Anesthesia. $10^{\text {th }}$ ed. WB Saunders, London, England.

Haskins SC, JD Patz, TB Farber. 1986. Xylazine and xylazine-ketamine in dogs. Am J Vet Res 47, 636-641. 
Havemann U, K Kuschinsky. 1981. Further characterization of opioid receptors in the striatum mediating muscular rigidity in rats. Naunyn Schmiedebergs Arch Pharmacol 317, 321-325.

Hsu WH, SV McNeel. 1983. Effect of yohimbine on xylazine-induced prolongation of gastrointestinal transit in dogs. J Am Vet Med Assoc 183, 297-300.

Hug CC, MR Murphy, EP Rigel, WA Olson. 1981. Pharmacokinetics of morphine injected intravenously into the anesthetized dog. Anesthesiology 54, 38-47.

Jarvis N, GC England. 1991. Reversal of xylazine sedation in dogs. Vet Rec 128, 323-325.

Jurna I, N Ruzdic, T Nell. 1972. The effect of alpha-methyl-p-tyrosine and substantia nigra lesions on spinal motor activity in the rat. Eur J Pharmacol 20, 341.

Klide AM, HW Calderwood, LR Soma. 1975. Cardiopulmonary effects of xylazine in dogs. Am J Vet Res 36, 931-935.

Ko JC, JE Bailey, LS Pablo, TG Heaton-Jones. 1996. Comparison of sedative and cardiorespiratory effects of medetomidine and medetomidinebutorphanol combination in dogs. Am J Vet Res 57, 535-540.

Kramer S, I Nolte, W Jöchle. 1996. Clinical comparison of medetomidine with xylazine/l-methadone in dogs. Vet Rec 138, 128-133.

Kuo WC, RD Keegan. 2004. Comparative cardiovascular, analgesic, and sedative effects of medetomidine, medetomidine-hydromorphone, and medetomidine-butorphanol in dogs. Am J Vet Res 65, 931-937.

Lascelles BDX. 2000. Clinical pharmacology of analgesic agents. In: Hellebrekers LJ (ed). Animal Pain. A practice-oriented approach to an effective pain control in animals. Van der wees, The Netherlands, Pp 85-116.

Leppänen MK, BC McKusick, MM Granholm, FC Westerholm, R Tulamo, CE Short. 2006. Clinical efficacy and safety of dexmedetomidine and buprenorphine, butorphanol or diazepam for canine hip radiography. J Small Anim Pract 47, 663-669.

Maze M, W Tranquilli. 1991. Alpha-2 adrenoceptor agonists: Defining the role in clinical anesthesia. Anesthesiology 74, 581-605.

Monteiro ER, CDN Figueroa, JC Choma, D Campagnol, C Bettini. 2008. Effects of methadone, alone or in combination with acepromazine or xylazine, on sedation and physiologic values in dogs. Vet Anaes Analg 35, 519-527.

Muir WW. 1991. Standing chemical restraint in horses. In: Muir WW, Hubbell JAE (eds). Equine Anesthesia. Monitoring and Emergency Therapy. Mosby, New York, USA.

Muir WW, JL Ford, GE Karpa, EE Harrison, JE Gadawski. 1999. Effects of intramuscular administration of low doses of medetomidine and medetomidine-butorphanol in middle-aged and old dogs. J Am Vet Med Assoc 215, 1116-1120.

Muir WW, JA Hubell, RT Skarda, RM Bednarski. 2001. Manual de Anestesia Veterinaria. $2^{\text {nd }}$ ed. Mosby, Madrid, España.

Muir WW. 2002. Drugs used to treat pain. In: Gaynor JS, Muir WW (eds). Handbook of Veterinary Pain Management. Mosby, St. Louis, USA.

Paddleford RR 1999. Preanesthetic Agents. Manual of Small Animal Anesthesia. $2^{\text {nd }}$ Ed. WB Saunders, Philadelphia, USA.

Plumb DC. 1999. Veterinary Drug Handbook. $3^{\text {rd }}$ ed. Iowa State University Press, Ames, USA.

Pypendop BH, JP Verstegen. 1998. Hemodynamic effects of medetomidine in the dog: a dose titration study. Vet Surg 27, 612-622.

Robinson KJ, RS Jones, PJ Cripps. 2001. Effects of medetomidine and buprenorphine administered for sedation in dogs. J Small Anim Pract 42, 444-447.

Sumano HS, L Ocampo. 1997. Farmacología Veterinaria. 2a ed. McGrawHill Interamericana, México D.F., México.

Thurmon JC, WJ Tranquilli, GJ Benson. 1996. Lumb \& Jones' Veterinary Anesthesia . $3^{\text {rd }}$ ed. Williams \& Wilkins, Baltimore, USA.

Tomizawa N, J Tomita, K Nakamura, S Hara. 1997. A comparative study of medetomidine-butorphanol-ketamine and medetomidine-ketamine anaesthesia in dogs. Zentralbl Veterinärmed A 44, 189-194.

Tyner CL, BJ Woody, JS Reid, EP Chafetz, HA Lederer, JF Norton, TJ Keefe, W Jöchle. 1997. Multicenter clinical comparison of sedative and analgesic effects of medetomidine and xylazine in dogs. J Am Vet Med Assoc 211, 1413-1417.

Young LE, JC Brearley, LS Richards, DH Bartram, RS Jones. 1990. Medetomidine as a premedicant in dogs and its reversal by atipamezole. J Small Anim Pract 31, 554-559. 